\title{
Research Article \\ Doob's Type Inequality and Strong Law of Large Numbers for Demimartingales
}

\author{
Wang Xuejun, Hu Shuhe, Zhao Ting, and Yang Wenzhi
}

School of Mathematical Science, Anhui University, Hefei 230039, China

Correspondence should be addressed to Hu Shuhe, hushuhe@263.net.com

Received 9 October 2009; Revised 14 December 2009; Accepted 28 January 2010

Academic Editor: Andrei Volodin

Copyright (C) 2010 Wang Xuejun et al. This is an open access article distributed under the Creative Commons Attribution License, which permits unrestricted use, distribution, and reproduction in any medium, provided the original work is properly cited.

We establish some maximal inequalities for demimartingales which generalize the result of Wang (2004). The maximal inequality for demimartingales is used as a key inequality to establish other results including Doob's type maximal inequality, strong law of large numbers, strong growth rate, and integrability of supremum for demimartingales, which generalize and improve partial results of Christofides (2000) and Prakasa Rao (2007).

\section{Introduction}

Definition 1.1. Let $S_{1}, S_{2}, \ldots$ be an $L^{1}$ sequence of random variables. Assume that for $j=$ $1,2, \ldots$

$$
E\left\{\left(S_{j+1}-S_{j}\right) f\left(S_{1}, \ldots, S_{j}\right)\right\} \geq 0
$$

for all coordinatewise nondecreasing functions $f$ such that the expectation is defined. Then $\left\{S_{j}, j \geq 1\right\}$ is called a demimartingale. If in addition the function $f$ is assumed to be nonnegative, then the sequence $\left\{S_{j}, j \geq 1\right\}$ is called a demisubmartingale.

Definition 1.2. A finite collection of random variables $X_{1}, X_{2}, \ldots, X_{m}$ is said to be associated if

$$
\operatorname{Cov}\left\{f\left(X_{1}, \ldots, X_{m}\right), g\left(X_{1}, \ldots, X_{m}\right)\right\} \geq 0
$$


for any two coordinatewise nondecreasing functions $f, g$ on $\mathbb{R}^{m}$ such that the covariance is defined. An infinite sequence $\left\{X_{n}, n \geq 1\right\}$ is associated if every finite subcollection is associated.

Definition 1.3. A finite collection of random variables $X_{1}, X_{2}, \ldots, X_{n}$ is said to be strongly positive dependent if

$$
P\left(\bar{X}_{1} \in \Lambda_{1} ; \bar{X}_{2} \in \Lambda_{2}\right) \geq P\left(\bar{X}_{1} \in \Lambda_{1}\right) P\left(\bar{X}_{2} \in \Lambda_{2}\right)
$$

for all Borel measurable and increasing (or decreasing) set pairs $\left(\Lambda_{1}, \Lambda_{2}\right) \subset R_{1} \times R_{2}$ (A set $\Lambda$ is said increasing (or decreasing) if $x \leq$ (or $\geq) y$ implies $y \in \Lambda$ for any $x \in \Lambda$ ), where

$$
\begin{gathered}
\bar{X}_{1}=\left(X_{i}, i \in I\right), \quad \bar{X}_{2}=\left(X_{i}, i \in I^{c}\right), \quad I \subset(1,2, \ldots, n), I^{c}=(1,2, \ldots, n) \backslash I, \\
R_{1}=\mathbb{R}^{|I|}, \quad R_{2}=\mathbb{R}^{\left|I^{c}\right|} \quad(|I| \text { stands for the base of } I) .
\end{gathered}
$$

An infinite sequence $\left\{X_{n}, n \geq 1\right\}$ is strongly positive dependent if every finite subcollection is strongly positive dependent.

Remark 1.4. Chow [1] proved a maximal inequality for submartingales. Newman and Wright [2] extended Doob's maximal inequality and upcrossing inequality to the case of demimartingales, and pointed out that the partial sum of a sequence of mean zero associated random variables is a demimartingale. Christofides [3] showed that the Chow's maximal inequality for (sub)martingales can be extended to the case of demi(sub)martingales. Wang [4] obtained Doob's type inequality for more general demimartingales. Hu et al. [5] gave a strong law of large numbers and growth rate for demimartingales. Prakasa Rao [6] established some maximal inequalities for demisubmartingales and N-demisupermartingales.

It is easily seen that the partial sum of a sequence of mean zero strongly positive dependent random variables is also a demimartingale by the inequality (3) in Zheng [7], that is, for all $n \geq 1$,

$$
E\left\{f\left(S_{1}, \ldots, S_{n}\right)\left(S_{n+1}-S_{n}\right)\right\}=E\left\{f\left(X_{1}, X_{1}+X_{2}, \ldots, X_{1}+X_{2}+\cdots+X_{n}\right) X_{n+1}\right\} \geq 0
$$

for all coordinatewise nondecreasing functions $f$ such that the expectation is defined. Therefore, the main results of this paper hold for the partial sums of sequences of mean zero associated random variables and strongly positive dependent random variables.

Let $\left\{X_{n}, n \geq 1\right\}$ and $\left\{S_{n}, n \geq 1\right\}$ be sequences of random variables defined on a fixed probability space $(\Omega, \mathcal{F}, P)$ and $I(A)$ the indicator function of the event $A$. Denote $S_{0}=0$, $X^{+}=\max (0, X), X^{-}=\max (0,-X), \log x=\log _{e} x=\ln x, \log ^{+} x=\ln (\max (x, 1))$. The main results of this paper depend on the following lemmas. 
Lemma 1.5 (see Wang [4, Theorem 2.1]). Let $\left\{S_{n}, n \geq 1\right\}$ be a demimartingale and g a nonnegative convex function on $\mathbb{R}$ with $g(0)=0$ and $g\left(S_{i}\right) \in L^{1}, i \geq 1$. Let $\left\{c_{k}, k \geq 1\right\}$ be a nonincreasing sequence of positive numbers. Then for any $\varepsilon>0$,

$$
\varepsilon P\left[\max _{1 \leq k \leq n} c_{k} g\left(S_{k}\right) \geq \varepsilon\right] \leq \sum_{j=1}^{n} c_{j} E\left[\left(g\left(S_{j}\right)-g\left(S_{j-1}\right)\right) I\left(\max _{1 \leq k \leq n} c_{k} g\left(S_{k}\right) \geq \varepsilon\right)\right] .
$$

Lemma 1.6 (see Fazekas and Klesov [8, Theorem 2.1] and Hu et al. [5, Lemma 1.5]). Let $\left\{X_{n}, n \geq 1\right\}$ be a random variable sequence and $S_{n}=\sum_{i=1}^{n} X_{i}$ for $n \geq 1$. Let $b_{1}, b_{2}, \ldots$ be a nondecreasing unbounded sequence of positive numbers and $\alpha_{1}, \alpha_{2}, \ldots$ nonnegative numbers. Let $p$ and $C$ be fixed positive numbers. Assume that for each $n \geq 1$,

$$
E\left(\max _{1 \leq l \leq n}\left|S_{l}\right|\right)^{p} \leq C \sum_{l=1}^{n} \alpha_{l}, \quad \sum_{l=1}^{\infty} \frac{\alpha_{l}}{b_{l}^{p}}<\infty
$$

then

$$
\lim _{n \rightarrow \infty} \frac{S_{n}}{b_{n}}=0 \quad \text { a.s., }
$$

and with the growth rate

$$
\frac{S_{n}}{b_{n}}=O\left(\frac{\beta_{n}}{b_{n}}\right) \text { a.s., }
$$

where

$$
\beta_{n}=\max _{1 \leq k \leq n} b_{k} v_{k}^{\delta / p}, \quad \forall 0<\delta<1, \quad v_{n}=\sum_{k=n}^{\infty} \frac{\alpha_{k}}{b_{k}^{p}}, \quad \lim _{n \rightarrow \infty} \frac{\beta_{n}}{b_{n}}=0
$$

In addition,

$$
E\left(\max _{1 \leq l \leq n}\left|\frac{S_{l}}{b_{l}}\right|\right)^{p} \leq 4 C \sum_{l=1}^{n} \frac{\alpha_{l}}{b_{l}^{p}}<\infty, \quad E\left(\sup _{l \geq 1}\left|\frac{S_{l}}{b_{l}}\right|\right)^{p} \leq 4 C \sum_{l=1}^{\infty} \frac{\alpha_{l}}{b_{l}^{p}}<\infty .
$$

If further assumes one that $\alpha_{n}>0$ for infinitely many $n$, then

$$
E\left(\sup _{l \geq 1}\left|\frac{S_{l}}{\beta_{l}}\right|\right)^{p} \leq 4 C \sum_{l=1}^{\infty} \frac{\alpha_{l}}{\beta_{l}^{p}}<\infty
$$

Lemma 1.7 (see Christofides [3, Lemma 2.1, Corollary 2.1]). (i) If $\left\{S_{n}, n \geq 1\right\}$ is a demisubmartingale (or a demimartingale) and $g$ is a nondecreasing convex function such that $g\left(S_{i}\right) \in$ $L^{1}, i \geq 1$, then $\left\{g\left(S_{n}\right), n \geq 1\right\}$ is a demisubmartingale.

(ii) If $\left\{S_{n}, n \geq 1\right\}$ is a demimartingale, then $\left\{S_{n}^{+}, n \geq 1\right\}$ is a demisubmartingale and $\left\{S_{n}^{-}, n \geq\right.$ $1\}$ is a demisubmartingale. 
Lemma 1.8 (see Hu et al. [9, Theorem 2.1]). Let $\left\{S_{n}, n \geq 1\right\}$ be a demimartingale and $\left\{c_{k}, k \geq 1\right\}$ be a nonincreasing sequence of positive numbers. Let $v \geq 1$ and $E\left|S_{k}\right|^{v}<\infty$ for each $k$, then for any $\varepsilon>0$ and $1 \leq n \leq N$,

$$
P\left\{\max _{n \leq k \leq N} c_{k}\left|S_{k}\right| \geq \varepsilon\right\} \leq \frac{2}{\varepsilon^{v}}\left\{c_{n}^{v} E\left|S_{n}\right|^{\nu}+\sum_{k=n+1}^{N} c_{k}^{v} E\left(\left|S_{k}\right|^{\nu}-\left|S_{k-1}\right|^{\nu}\right)\right\} .
$$

Lemma 1.9 (see Christofides [3, Corollary 2.4, Theorem 2.1]). (i) Let $\left\{S_{n}, n \geq 1\right\}$ be a demisubmartingale. Then for any $\varepsilon>0$,

$$
\varepsilon P\left(\max _{1 \leq k \leq n} S_{k} \geq \varepsilon\right) \leq \int_{\left(\max _{1 \leq k \leq n} S_{k} \geq \varepsilon\right)} S_{n} d P .
$$

(ii) Let $\left\{S_{n}, n \geq 1\right\}$ be a demisubmartingale and $\left\{c_{k}, k \geq 1\right\}$ a nonincreasing sequence of positive numbers. Then for any $\varepsilon>0$,

$$
\varepsilon P\left(\max _{1 \leq k \leq n} c_{k} S_{k} \geq \varepsilon\right) \leq \sum_{j=1}^{n} c_{j} E\left(S_{j}^{+}-S_{j-1}^{+}\right)
$$

Using Lemma 1.5, Wang [4] obtained the following inequalities for demimartingales.

Theorem 1.10 (see Wang [4, Corollary 2.1]). Let $\left\{S_{n}, n \geq 1\right\}$ be a demimartingale and $\left\{c_{k}, k \geq 1\right\}$ a nonincreasing sequence of positive numbers. Then

$$
\begin{gathered}
E\left[\max _{1 \leq k \leq n} c_{k}\left|S_{k}\right|\right]^{p} \leq\left(\frac{p}{p-1}\right)^{p} E\left[\sum_{j=1}^{n} c_{j}\left(\left|S_{j}\right|-\left|S_{j-1}\right|\right)\right]^{p}, \quad p>1 \\
E\left[\max _{1 \leq k \leq n} c_{k}\left|S_{k}\right|\right] \leq \frac{e}{e-1}\left\{1+E\left[\left(\sum_{j=1}^{n} c_{j}\left(\left|S_{j}\right|-\left|S_{j-1}\right|\right)\right) \log ^{+}\left(\sum_{j=1}^{n} c_{j}\left(\left|S_{j}\right|-\left|S_{j-1}\right|\right)\right)\right]\right\} .
\end{gathered}
$$

We point out that there is a mistake in the proof of (1.17), that is,

$$
E\left[\max _{1 \leq j \leq n} c_{j}\left|S_{j}\right|\right]-1 \leq E\left[\left(\sum_{j=1}^{n} c_{j}\left(\left|S_{j}\right|-\left|S_{j-1}\right|\right)\right) \log \left(\max _{1 \leq j \leq n} c_{j}\left|S_{j}\right|\right)\right]
$$

should be replaced by

$$
E\left[\max _{1 \leq j \leq n} c_{j}\left|S_{j}\right|\right]-1 \leq E\left[\left(\sum_{j=1}^{n} c_{j}\left(\left|S_{j}\right|-\left|S_{j-1}\right|\right)\right) \log ^{+}\left(\max _{1 \leq j \leq n} c_{j}\left|S_{j}\right|\right)\right] .
$$


In fact, by Lemma 1.5 and Fubini Theorem, we can see that

$$
\begin{aligned}
E\left[\max _{1 \leq j \leq n} c_{j}\left|S_{j}\right|\right]-1 & \leq \int_{1}^{\infty} P\left(\max _{1 \leq j \leq n} c_{j}\left|S_{j}\right| \geq x\right) d x \\
& \leq \int_{1}^{\infty} \frac{1}{x} E\left[\sum_{j=1}^{n} c_{j}\left(\left|S_{j}\right|-\left|S_{j-1}\right|\right) I\left(\max _{1 \leq j \leq n} c_{j}\left|S_{j}\right| \geq x\right)\right] d x \\
& \leq E\left[\left(\sum_{j=1}^{n} c_{j}\left(\left|S_{j}\right|-\left|S_{j-1}\right|\right)\right) \log ^{+}\left(\max _{1 \leq j \leq n} c_{j}\left|S_{j}\right|\right)\right] .
\end{aligned}
$$

The rest of the proof is similar to Corollary 2.1 in Wang [4].

The same problem exists in Shiryaev [10, page 495, in the proof of Theorem 2] and Krishna and Soumendra [11, page 414, in the proof of Theorem 13.2.13]. For example, the following inequality

$$
E\left(\max _{0 \leq j \leq n}\left|X_{j}\right|\right)-1 \leq E\left(X_{n} \int_{0}^{\max _{0 \leq j}\left|X_{j}\right|-1} \frac{d t}{1+t}\right)=E\left[X_{n} \log \left(\max _{0 \leq j \leq n}\left|X_{j}\right|\right)\right]
$$

in Shiryaev [10, page 495] should be revised as

$$
\begin{aligned}
E\left(\max _{0 \leq j \leq n}\left|X_{j}\right|\right)-1 & \leq E\left[X_{n} I\left(\max _{0 \leq j \leq n}\left|X_{j}\right| \geq 1\right) \int_{0}^{\max \left|X_{j}\right|-1} \frac{d t}{1+t}\right] \\
& =E\left[X_{n} \log ^{+}\left(\max _{0 \leq j \leq n}\left|X_{j}\right|\right)\right] .
\end{aligned}
$$

\section{Main Results and Their Proofs}

Theorem 2.1. Let $\left\{S_{n}, n \geq 1\right\}$ be a demimartingale and $g$ a nonnegative convex function on $\mathbb{R}$ with $g(0)=0$. Let $\left\{c_{k}, k \geq 1\right\}$ be a nonincreasing sequence of positive numbers. $p>1$. Suppose that $E\left(g\left(S_{k}\right)\right)^{p}<\infty$ for each $k \geq 1$, then for every $n \geq 1$,

$$
\begin{gathered}
E\left[\max _{1 \leq k \leq n} c_{k} g\left(S_{k}\right)\right]^{p} \leq\left(\frac{p}{p-1}\right)^{p} E\left[\sum_{j=1}^{n} c_{j}\left(g\left(S_{j}\right)-g\left(S_{j-1}\right)\right)\right]^{p} \\
E\left[\max _{1 \leq k \leq n} c_{k} g\left(S_{k}\right)\right] \leq \frac{e}{e-1}\left\{1+E\left[\left(\sum_{j=1}^{n} c_{j}\left(g\left(S_{j}\right)-g\left(S_{j-1}\right)\right)\right) \log ^{+}\left(\sum_{j=1}^{n} c_{j}\left(g\left(S_{j}\right)-g\left(S_{j-1}\right)\right)\right)\right]\right\} .
\end{gathered}
$$


Proof. By Lemma 1.5 and Hölder's inequality, we have

$$
\begin{aligned}
E\left[\max _{1 \leq k \leq n} c_{k} g\left(S_{k}\right)\right]^{p} & =p \int_{0}^{\infty} x^{p-1} P\left[\max _{1 \leq k \leq n} c_{k} g\left(S_{k}\right) \geq x\right] d x \\
& \leq p \int_{0}^{\infty} x^{p-2} E\left[\sum_{j=1}^{n} c_{j}\left(g\left(S_{j}\right)-g\left(S_{j-1}\right)\right) I\left(\max _{1 \leq k \leq n} c_{k} g\left(S_{k}\right) \geq x\right)\right] d x \\
& =\frac{p}{p-1} E\left\{\left[\sum_{j=1}^{n} c_{j}\left(g\left(S_{j}\right)-g\left(S_{j-1}\right)\right)\right]\left(\max _{1 \leq k \leq n} c_{k} g\left(S_{k}\right)\right)^{p-1}\right\} \\
& \leq \frac{p}{p-1}\left\{E\left[\sum_{j=1}^{n} c_{j}\left(g\left(S_{j}\right)-g\left(S_{j-1}\right)\right)\right]^{p}\left\{E\left[\max _{1 \leq k \leq n} c_{k} g\left(S_{k}\right)\right]^{p}\right\}^{1 / p},\right.
\end{aligned}
$$

where $q$ is a real number and satisfies $1 / p+1 / q=1$. Since $E\left(g\left(S_{k}\right)\right)^{p}<\infty$ for each $k \geq 1$, we can obtain

$$
\left\{E\left[\max _{1 \leq k \leq n} c_{k} g\left(S_{k}\right)\right]^{p}\right\}^{1 / p} \leq \frac{p}{p-1}\left\{E\left[\sum_{j=1}^{n} c_{j}\left(g\left(S_{j}\right)-g\left(S_{j-1}\right)\right)\right]^{p}\right\}^{1 / p}
$$

therefore,

$$
E\left[\max _{1 \leq k \leq n} c_{k} g\left(S_{k}\right)\right]^{p} \leq\left(\frac{p}{p-1}\right)^{p} E\left[\sum_{j=1}^{n} c_{j}\left(g\left(S_{j}\right)-g\left(S_{j-1}\right)\right)\right]^{p}
$$

Similar to the proof of (2.3) and using Lemma 1.5 again, we can see that

$$
E\left[\max _{1 \leq k \leq n} c_{k} g\left(S_{k}\right)\right] \leq 1+E\left[\left(\sum_{j=1}^{n} c_{j}\left(g\left(S_{j}\right)-g\left(S_{j-1}\right)\right)\right) \log ^{+}\left(\max _{1 \leq k \leq n} c_{k} g\left(S_{k}\right)\right)\right] .
$$

For constants $a \geq 0$ and $b>0$, it follows that

$$
a \log ^{+} b \leq a \log ^{+} a+b e^{-1} .
$$


Combining (2.6) and (2.7), we have

$$
\begin{aligned}
E\left[\max _{1 \leq k \leq n} c_{k} g\left(S_{k}\right)\right] \leq 1 & +E\left[\left(\sum_{j=1}^{n} c_{j}\left(g\left(S_{j}\right)-g\left(S_{j-1}\right)\right)\right) \log ^{+}\left(\sum_{j=1}^{n} c_{j}\left(g\left(S_{j}\right)-g\left(S_{j-1}\right)\right)\right)\right] \\
& +e^{-1} E\left[\max _{1 \leq k \leq n} c_{k} g\left(S_{k}\right)\right] .
\end{aligned}
$$

Thus, (2.2) follows from (2.8) immediately. The proof is complete.

Remark 2.2. If we take $g(x)=|x|$ in Theorem 2.1, then Theorem 2.1 implies Corollary 2.1 in Wang [4].

Corollary 2.3. Let the conditions of Theorem 2.1 be satisfied with $c_{k} \equiv 1$ for each $k \geq 1$. Then for every $n \geq 1$,

$$
\begin{gathered}
E\left[\max _{1 \leq k \leq n} g\left(S_{k}\right)\right]^{p} \leq\left(\frac{p}{p-1}\right)^{p} E\left(g\left(S_{n}\right)\right)^{p}, \\
E\left[\max _{1 \leq k \leq n} g\left(S_{k}\right)\right] \leq \frac{e}{e-1}\left\{1+E\left[g\left(S_{n}\right) \log ^{+} g\left(S_{n}\right)\right]\right\} .
\end{gathered}
$$

Corollary 2.4 (Doob's type maximal inequality for demimartingales). Let $p>1$ and $\left\{S_{n}, n \geq\right.$ $1\}$ be a demimartingale. Suppose that $E\left|S_{k}\right|^{p}<\infty$ for each $k \geq 1$, then for every $n \geq 1$,

$$
\begin{gathered}
E\left[\max _{1 \leq k \leq n}\left|S_{k}\right|\right]^{p} \leq\left(\frac{p}{p-1}\right)^{p} E\left|S_{n}\right|^{p}, \\
E\left[\max _{1 \leq k \leq n}\left|S_{k}\right|\right] \leq \frac{e}{e-1}\left[1+E\left(\left|S_{n}\right| \log ^{+}\left|S_{n}\right|\right)\right] .
\end{gathered}
$$

Theorem 2.5. Let $\left\{S_{n}, n \geq 1\right\}$ be a demimartingale and $g$ a nonnegative convex function on $\mathbb{R}$ with $g(0)=0$. Let $\left\{b_{n}, n \geq 1\right\}$ be a nondecreasing unbounded sequence of positive numbers. $p>1$. Suppose that $E\left(g\left(S_{k-1}\right)\right)^{p} \leq E\left(g\left(S_{k}\right)\right)^{p}<\infty$ for each $k \geq 1$ and

$$
\sum_{n=1}^{\infty} \frac{E\left(g\left(S_{n}\right)\right)^{p}-E\left(g\left(S_{n-1}\right)\right)^{p}}{b_{n}^{p}}<\infty
$$

then $\lim _{n \rightarrow \infty}\left(g\left(S_{n}\right) / b_{n}\right)=0$ a.s., and (1.9)-(1.10) hold $\left(S_{n}\right.$ is replaced by $\left.g\left(S_{n}\right)\right)$, where

$$
\alpha_{n}=\left(\frac{p}{p-1}\right)^{p}\left[E\left(g\left(S_{n}\right)\right)^{p}-E\left(g\left(S_{n-1}\right)\right)^{p}\right], \quad n \geq 1
$$


In addition,

$$
E\left(\max _{1 \leq k \leq n}\left|\frac{g\left(S_{k}\right)}{b_{k}}\right|^{p}\right) \leq 4 \sum_{k=1}^{n} \frac{\alpha_{k}}{b_{k}^{p}}<\infty, \quad E\left(\sup _{n \geq 1}\left|\frac{g\left(S_{n}\right)}{b_{n}}\right|^{p}\right) \leq 4 \sum_{n=1}^{\infty} \frac{\alpha_{n}}{b_{n}^{p}}<\infty .
$$

If further one assumes that $\alpha_{k}>0$ for infinitely many $k$, then

$$
E\left(\sup _{n \geq 1}\left|\frac{g\left(S_{n}\right)}{\beta_{n}}\right|^{p}\right) \leq 4 \sum_{n=1}^{\infty} \frac{\alpha_{n}}{\beta_{n}^{p}}<\infty
$$

Proof. By the condition of the theorem, we can see that $\alpha_{n} \geq 0$ for all $n \geq 1$. Thus,

$$
E\left[\max _{1 \leq k \leq n} g\left(S_{k}\right)\right]^{p} \leq\left(\frac{p}{p-1}\right)^{p} E\left(g\left(S_{n}\right)\right)^{p}=\sum_{k=1}^{n} \alpha_{k}
$$

follows from (2.9) for each $n \geq 1$. By (2.12), we have

$$
\sum_{n=1}^{\infty} \frac{\alpha_{n}}{b_{n}^{p}}=\left(\frac{p}{p-1}\right)^{p} \sum_{n=1}^{\infty} \frac{E\left(g\left(S_{n}\right)\right)^{p}-E\left(g\left(S_{n-1}\right)\right)^{p}}{b_{n}^{p}}<\infty .
$$

Therefore, $\lim _{n \rightarrow \infty}\left(g\left(S_{n}\right) / b_{n}\right)=0$ a.s. follows from Lemma 1.6, (2.16), and (2.17); (1.9), (1.10), (2.14), (2.15) hold. This completes the proof of the theorem.

In Theorem 2.5, if we assume that $g(x)$ is a nonnegative and nondecreasing convex function on $\mathbb{R}$ with $g(0)=0$, then the condition " $E\left(g\left(S_{k-1}\right)\right)^{p} \leq E\left(g\left(S_{k}\right)\right)^{p}$ for each $k \geq 1$ " is satisfied.

Remark 2.6. Theorem 2.5 generalizes and improves the results of Theorem 2.2 in Christofides [3] and Theorem 2.7 in Prakasa Rao [6].

Theorem 2.7. Let $p>1$ and $\left\{S_{n}, n \geq 1\right\}$ a demimartingale with $E\left|S_{k}\right|^{p}<\infty$ for each $k \geq 1$. Let $\left\{b_{n}, n \geq 1\right\}$ be a nondecreasing sequence of positive numbers. If

$$
\sum_{k=1}^{\infty} \frac{E\left|S_{k}\right|^{p}-E\left|S_{k-1}\right|^{p}}{b_{k}^{p}}<\infty
$$

then for any $0<r<p$,

$$
E\left(\sup _{k \geq 1}\left|\frac{S_{k}}{b_{k}}\right|^{r}\right) \leq 1+\frac{2 r}{p-r} \sum_{k=1}^{\infty} \frac{E\left|S_{k}\right|^{p}-E\left|S_{k-1}\right|^{p}}{b_{k}^{p}}<\infty .
$$


Proof. Taking $n=1, v=p$ and $c_{k}=1 / b_{k}$ in Lemma 1.8, we have

$$
P\left\{\max _{1 \leq k \leq N}\left|\frac{S_{k}}{b_{k}}\right| \geq \varepsilon\right\} \leq \frac{2}{\varepsilon^{p}} \sum_{k=1}^{N} \frac{E\left|S_{k}\right|^{p}-E\left|S_{k-1}\right|^{p}}{b_{k}^{p}} .
$$

Thus, by (2.20) and (2.18), we can get

$$
\begin{aligned}
E\left(\sup _{k \geq 1}\left|\frac{S_{k}}{b_{k}}\right|^{r}\right) & \leq 1+\int_{1}^{\infty} P\left(\sup _{k \geq 1}\left|\frac{S_{k}}{b_{k}}\right|^{r}>t\right) d t=1+\int_{1}^{\infty} \lim _{N \rightarrow \infty} P\left(\max _{1 \leq k \leq N}\left|\frac{S_{k}}{b_{k}}\right|>t^{1 / r}\right) d t \\
& \leq 1+\frac{2 r}{p-r} \sum_{k=1}^{\infty} \frac{E\left|S_{k}\right|^{p}-E\left|S_{k-1}\right|^{p}}{b_{k}^{p}}<\infty .
\end{aligned}
$$

Theorem 2.8. Let $\left\{S_{n}, n \geq 1\right\}$ be a demisubmartingale and $g$ a nondecreasing and nonnegative convex function on $\mathbb{R}$ with $g(0)=0$ and $g\left(S_{i}\right) \in L^{1}, i \geq 1$. Let $\left\{c_{k}, k \geq 1\right\}$ be a nonincreasing sequence of positive numbers. Then for all $0<p<1$ and each $n \geq 1$,

$$
E\left[\max _{1 \leq k \leq n} c_{k} g\left(S_{k}\right)\right]^{p} \leq\left(c_{n} E g\left(S_{n}\right)\right)^{p-1}\left[c_{n} E g\left(S_{n}\right)+\frac{p}{1-p} \sum_{j=1}^{n} c_{j} E\left(g\left(S_{j}\right)-g\left(S_{j-1}\right)\right)\right] .
$$

Proof. By Fubini theorem, it is easy to check that

$$
\begin{aligned}
E\left[\max _{1 \leq k \leq n} c_{k} g\left(S_{k}\right)\right]^{p} & =p \int_{0}^{\infty} x^{p-1} P\left(\max _{1 \leq k \leq n} c_{k} g\left(S_{k}\right) \geq x\right) d x \\
& \leq p \int_{0}^{c_{n} E g\left(S_{n}\right)} x^{p-1} d x+p \int_{c_{n} E g\left(S_{n}\right)}^{\infty} x^{p-1} P\left(\max _{1 \leq k \leq n} c_{k} g\left(S_{k}\right) \geq x\right) d x \\
& =\left(c_{n} E g\left(S_{n}\right)\right)^{p}+p \int_{c_{n} E g\left(S_{n}\right)}^{\infty} x^{p-1} P\left(\max _{1 \leq k \leq n} c_{k} g\left(S_{k}\right) \geq x\right) d x .
\end{aligned}
$$

It follows from Lemma 1.7(i) and Lemma 1.9(ii) that

$$
\begin{aligned}
p \int_{c_{n} E g\left(S_{n}\right)}^{\infty} x^{p-1} P\left(\max _{1 \leq k \leq n} c_{k} g\left(S_{k}\right) \geq x\right) d x & \leq p \int_{c_{n} E g\left(S_{n}\right)}^{\infty} x^{p-2} \sum_{j=1}^{n} c_{j} E\left(g\left(S_{j}\right)-g\left(S_{j-1}\right)\right) d x \\
& =\frac{p}{1-p}\left(c_{n} E g\left(S_{n}\right)\right)^{p-1} \sum_{j=1}^{n} c_{j} E\left(g\left(S_{j}\right)-g\left(S_{j-1}\right)\right) .
\end{aligned}
$$

Therefore, (2.22) follows from the above statements immediately. 
Corollary 2.9. Let the conditions of Theorem 2.8 be satisfied with $c_{k} \equiv 1$ for each $k \geq 1$. Then for all $0<p<1$ and each $n \geq 1$,

$$
E\left[\max _{1 \leq k \leq n} g\left(S_{k}\right)\right]^{p} \leq \frac{1}{1-p}\left(E g\left(S_{n}\right)\right)^{p}
$$

By Corollary 2.9, we can get the following theorem.

Theorem 2.10. Let $\left\{S_{n}, n \geq 1\right\}$ be a demisubmartingale and $g$ a nondecreasing and nonnegative convex function on $\mathbb{R}$ with $g(0)=0$ and $g\left(S_{i}\right) \in L^{1}, i \geq 1$. Let $\left\{b_{n}, n \geq 1\right\}$ be a nondecreasing unbounded sequence of positive numbers. If there exists some $0<p<1$ such that

$$
\sum_{n=1}^{\infty} \frac{\left(E g\left(S_{n}\right)\right)^{p}-\left(E g\left(S_{n-1}\right)\right)^{p}}{b_{n}^{p}}<\infty
$$

then $\lim _{n \rightarrow \infty}\left(g\left(S_{n}\right) / b_{n}\right)=0$ a.s., and (1.9)-(1.10) hold $\left(S_{n}\right.$ is replaced by $\left.g\left(S_{n}\right)\right)$, where

$$
\alpha_{n}=\frac{1}{1-p}\left[\left(E g\left(S_{n}\right)\right)^{p}-\left(E g\left(S_{n-1}\right)\right)^{p}\right], \quad n \geq 1 .
$$

In addition,

$$
E\left(\max _{1 \leq k \leq n}\left|\frac{g\left(S_{k}\right)}{b_{k}}\right|^{p}\right) \leq 4 \sum_{k=1}^{n} \frac{\alpha_{k}}{b_{k}^{p}}<\infty, \quad E\left(\sup _{n \geq 1}\left|\frac{g\left(S_{n}\right)}{b_{n}}\right|^{p}\right) \leq 4 \sum_{n=1}^{\infty} \frac{\alpha_{n}}{b_{n}^{p}}<\infty .
$$

If further one assumes that $\alpha_{k}>0$ for infinitely many $k$, then

$$
E\left(\sup _{n \geq 1}\left|\frac{g\left(S_{n}\right)}{\beta_{n}}\right|^{p}\right) \leq 4 \sum_{n=1}^{\infty} \frac{\alpha_{n}}{\beta_{n}^{p}}<\infty
$$

Similar to the proof of Theorem 2.8 and using Lemma 1.9(i), we can get the following.

Theorem 2.11. Let $\left\{S_{n}, n \geq 1\right\}$ be a nonnegative demisubmartingale. Then for all $0<p<1$, $E\left[\max _{1 \leq k \leq n} S_{k}\right]^{p} \leq(1 /(1-p))\left(E S_{n}\right)^{p}$.

\section{Acknowledgments}

The authors are most grateful to the Editor Andrei Volodin and an anonymous referee for the careful reading of the manuscript and valuable suggestions which helped in significantly improving an earlier version of this paper. This work was supported by the National Natural Science Foundation of China (Grant no. 10871001, 60803059), Talents Youth Fund of Anhui Province Universities (Grant no. 2010SQRL016ZD), Youth Science Research Fund of Anhui University (Grant no. 2009QN011A), Provincial Natural Science Research Project of Anhui Colleges and the Innovation Group Foundation of Anhui University. 


\section{References}

[1] Y. S. Chow, "A martingale inequality and the law of large numbers," Proceedings of the American Mathematical Society, vol. 11, pp. 107-111, 1960.

[2] C. M. Newman and A. L. Wright, "Associated random variables and martingale inequalities," Zeitschrift für Wahrscheinlichkeitstheorie und Verwandte Gebiete, vol. 59, no. 3, pp. 361-371, 1982.

[3] T. C. Christofides, "Maximal inequalities for demimartingales and a strong law of large numbers," Statistics \& Probability Letters, vol. 50, no. 4, pp. 357-363, 2000.

[4] J. F. Wang, "Maximal inequalities for associated random variables and demimartingales," Statistics $\mathcal{E}$ Probability Letters, vol. 66, no. 3, pp. 347-354, 2004.

[5] S. H. Hu, G. J. Chen, and X. J. Wang, "On extending the Brunk-Prokhorov strong law of large numbers for martingale differences," Statistics E Probability Letters, vol. 78, no. 18, pp. 3187-3194, 2008.

[6] B. L. S. Prakasa Rao, "On some maximal inequalities for demisubmartingales and $N$-demisuper martingales," Journal of Inequalities in Pure and Applied Mathematics, vol. 8, no. 4, article 112, pp. 1$17,2007$.

[7] Y. H. Zheng, "Inequalities of moment and convergence theorem of order statistics of partial sums for a class of strongly positive dependent stochastic sequence," Acta Mathematicae Applicatae Sinica, vol. 24, no. 2, pp. 168-176, 2001 (Chinese).

[8] I. Fazekas and O. Klesov, "A general approach rate to the strong law of large numbers," Statistics $\mathcal{E}$ Probability Letters, vol. 45, no. 3, pp. 436-449, 2001.

[9] S. H. Hu, X. J. Wang, W. Z. Yang, and T. Zhao, "The Hàjek-Rènyi-type inequality for associated random variables," Statistics E Probability Letters, vol. 79, no. 7, pp. 884-888, 2009.

[10] A. N. Shiryaev, Probability, vol. 95 of Graduate Texts in Mathematics, Springer, New York, NY, USA, 2nd edition, 1996

[11] K. B. Athreya and S. N. Lahiri, Measure Theory and Probability Theory, Springer Texts in Statistics, Springer, New York, NY, USA, 2006. 